\title{
Etude Ethnobotanique De Quelques Espèces Du Genre Corchorus Rencontrées En Côte d'Ivoire
}

\author{
Ta Bi Irié Honoré \\ N'Guessan Koffi
}

Université Félix Houphouët-Boigny (Côte-d’Ivoire), U.F.R. Biosciences, Laboratoire de Botanique, Abidjan, Côte d'Ivoire

\section{Bomisso Edson Lezin}

Université Félix Houphouët-Boigny (Côte-d’Ivoire), U.F.R. Biosciences, Laboratoire de Physiologie Végétale, Abidjan, Côte d'Ivoire

\section{Assa Rebecca Rachel}

Université Félix Houphouët-Boigny (Côte-d’Ivoire), U.F.R. Biosciences, Laboratoire de Biochimie et Sciences des Aliments, Abidjan, Côte d'Ivoire

\section{Aké Séverin}

Université Félix Houphouët-Boigny (Côte-d’Ivoire), U.F.R. Biosciences, Laboratoire de Physiologie Végétale, Abidjan, Côte d'Ivoire

\section{doi: 10.19044/esj.2016.v12n24p415 URL:http://dx.doi.org/10.19044/esj.2016.v12n24p415}

\begin{abstract}
To contribute to the valorization of Corchorus plants, fifteen (15) communities in Côte d'Ivoire have been visited and 432 participants were selected, during an ethnobotanical survey. This survey helped us to identify four (4) different species of Corchorus which are: C. aestuans, C. lobatus, C. olitorius and $C$. tridens. These different species are grown by farmers in general and also by farmers of vegetables and foods crops. In Côte d'Ivoire these plants are called "Kroala". Kroala is the name given by Baoulé people from the centre part of Côte d'Ivoire. Krola can be used as main ingredient in the cooking of soups and it can also be used as medicinal plant. At food level, the leaves of different species of Corchorus are used in the cooking of soups for several meals throughout the country. At ethnomedicinal level, these different plant species are used in the treatment of several pathologies such as anemia, constipation... For instance, leaves, roots, leafy stems and seeds of these plant species are used to make several monospecific drugs recipes. For the benefits brought by Corchorus plants to people, the growing of these plants must be encouraged throughout the country.
\end{abstract}

Keywords: Corchorus, Côte d’Ivoire, Ethnobotany 


\section{Résumé}

Pour contribuer à la valorisation des plantes du genre Corchorus, 15 localités de la Côte d'Ivoire ont été visitées et 432 personnes ont été rencontrées, au cours d'une enquête ethnobotanique. Ces investigations ont permis d'identifier 4 espèces appartenant au genre Corchorus: $C$. aestuans, $C$. lobatus, $C$. olitorius et $C$. tridens. Ces espèces sont cultivées à la fois par les paysans et les maraîchers. En Côte d'Ivoire, ces plantes sont nommées «Kroala » qui est une appellation propre au groupe ethnique Baoulé localisé au centre du pays. Kroala est utilisé à la fois comme plante comestible et médicinale. Au plan alimentaire, les feuilles de ces espèces du genre Corchorus sont consommées sur l'étendue du territoire en sauce accompagnant plusieurs mets. Au plan ethnomedicinal, ces espèces interviennent dans le traitement de plusieurs pathologies telles que l'anémie, la constipation...Diverses parties de ces plantes sont utilisées à cet effet : les feuilles, les racines, les tiges feuillées et les graines, pour préparer plusieurs recettes médicamenteuses monospécifiques. Pour leurs importants apports aux populations, la culture de ces plantes doit être encouragée dans le pays.

Mots clés: Corchorus, Côte d’Ivoire, Ethnobotanique,

\section{INTRODUCTION}

Les connaissances actuelles situent le début de l'humanité à sept millions d’années environ (Guy, 2009). Depuis ce temps, l’homme a utilisé les plantes à diverses fins : artisanat, chasse de gibier, construction d'habitat, cosmétique, nourriture, pêche, zootechnie et soins de santé (N'Guessan, 2008). Parmi les nombreuses plantes utilisées par l'homme se trouvent les espèces du genre Corchorus. Plusieurs espèces répertoriées de ce genre sont utilisées comme plantes alimentaires dans certains pays d'Afrique de l'Ouest. Des travaux de Recherche menés sur quelques unes de ces plantes ont mis en exergue leurs nombreuses vertus thérapeutiques (Aiyeroja et Bello, 2006). Ainsi, les tiges feuillées de Chorchorus tridens sont utilisées dans la lutte contre la diarrhée, la toux et la blennorragie (Mbaye, 2002). Les feuilles de Corchorus olitorius sont indiquées contre la constipation et les carries dentaires alors que les graines de Corchorus aestuans interviennent dans le traitement de la colique et des pneumonies (Osawaru et al., 2012). En Côte d'Ivoire la consommation des plantes du genre Corchorus est très répandue (Aké et al. 2015, N’Guessan et al. 2015). Cependant les études orientées sur l'inventaire de ces espèces et surtout l'indication de leurs propriétés thérapeutiques sont fragmentaires. Cette étude répond à cette 
préoccupation. Elle a pour objectif d'identifier les plantes appartenant au genre Corchorus consommées en Côte d'Ivoire et d'indiquer leurs vertus thérapeutiques.

\section{MATÉRIEL ET MÉTHODES}

\section{Présentation du milieu d'étude}

L'enquête ethnobotanique s'est réalisée dans 15 localités de la Côte d'Ivoire (Figure 1). La Côte d'Ivoire est un pays de l'Afrique occidentale situé entre $4^{\circ} 30^{\prime}$ et $10^{\circ} 30^{\prime}$ de latitude Nord et entre $2^{\circ} 30^{\prime}$ et $8^{\circ} 30^{\prime}$ de latitude Ouest. Le pays s'étend sur une superficie de $322.462 \mathrm{Km}^{2}$. Il est limité au Nord par le Burkina Faso et le Mali, à l'Est par le Ghana, à l'Ouest par le Libéria et la Guinée et au Sud par le Golfe de Guinée. La population du pays est de 30 millions d'habitants RGPH (2014). Pendant cette étude, les investigations et la récolte des différents spécimens se sont déroulés dans les localités ivoiriennes suivantes :

- Abidjan, Bingerville au Sud ;

-Yamoussoukro, Bouaké, Katiola et Korhogo au Centre et CentreNord ;

-Abengourou, Bondoukou et Bouna à l'Est et Nord-Est ;

-Gagnoa, Daloa, Guiglo, Man, Touba et Odienné à l'ouest et au Nord-Ouest.

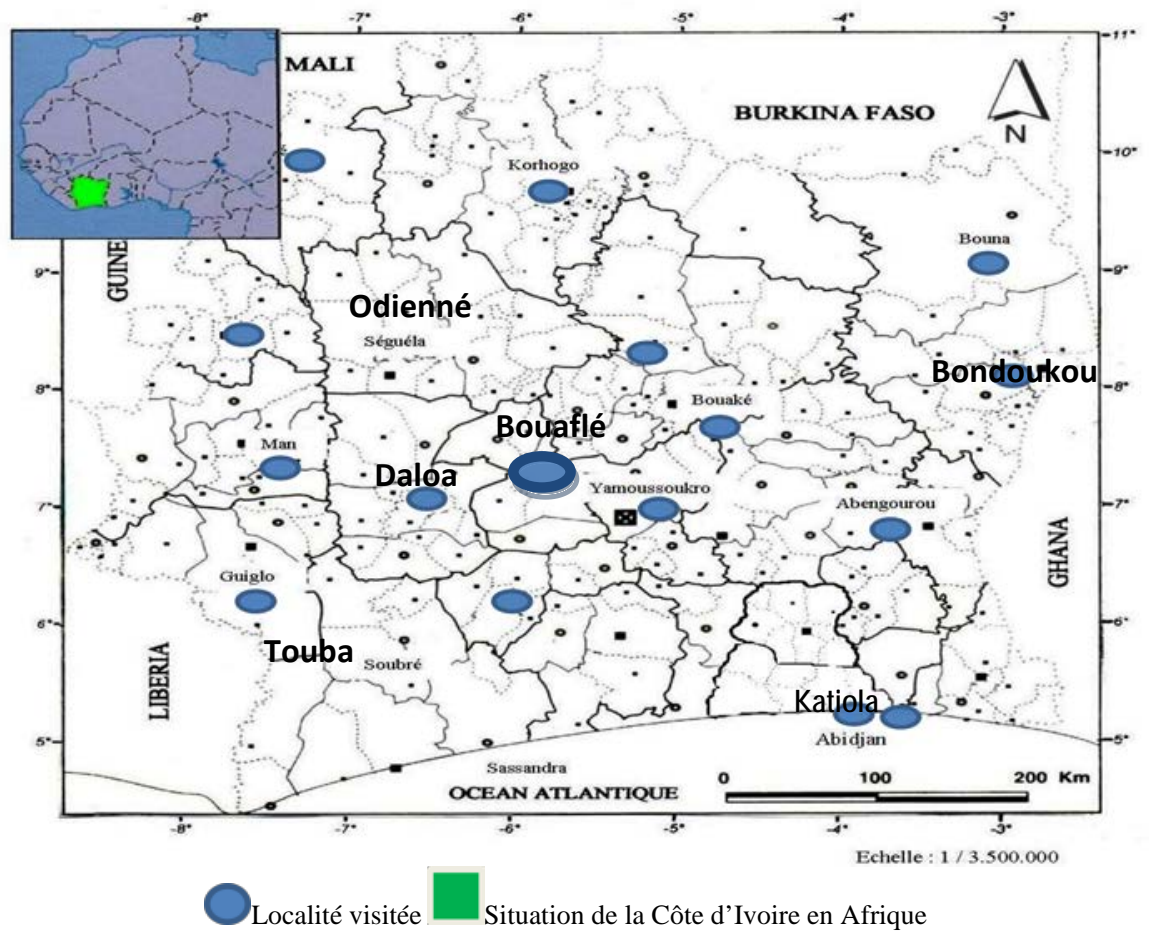

Figure 1 : Carte de localisation des sites visitées

Source : CEDA, 2001 modifié par N’Guessan et TA BI 


\section{Matériel}

\section{Matériel technique}

Le matériel technique pour les investigations ethnobotaniques se compose de: une fiche d'enquête pour la collecte des informations, un sécateur pour la récolte d’échantillons de spécimen, des sachets en matière plastique, du carton et des anciens journaux pour la conservation des échantillons. Un carnet de notes a été nécessaire pour l'enregistrement des informations complémentaires. Les prises de vue ont été réalisées à l'aide d'un appareil photo numérique de type SAMSUNG (16.2 méga pixels). Un ordinateur portable a été utile pour la saisie et l'analyse des données recueillies.

\section{Matériel végétal}

Le matériel végétal regroupe l'ensemble des espèces du genre Corchorus recensées dans les marchés, dans les maraichers et dans les champs, au niveau des différents sites visités.

\section{Méthode d'étude}

\section{Collecte de données ethnobotaniques et identification}

La collecte des données ethnobotaniques s'est faite selon des interrogations directes (Tahri et al., 2012 ; Doh, 2015) et des interrogations semi-directes nécessitant l'intervention d'un guide qui a parfois joué le rôle de traducteur. Les personnes interrogées ont été choisies selon leur domaine d'activité (vendeuses de plantes alimentaires, maraîchers et paysans). Ces différentes investigations ont été guidées par une fiche d’enquête. Ces échanges ont eu lieu durant deux périodes : de juin 2013 à août 2013 et de février 2015 à octobre 2015. Les différentes localités visitées ont été choisies selon leur situation géographique pour une bonne répartition de sites sur l'ensemble du territoire. Les marchés ont été choisis selon la taille et l'approvisionnement en plantes alimentaires. Les sites maraichers et les champs ont été choisis pour leur proximité. L’identification des échantillons a été faite au Centre National de Floristique (C.N.F) de l’Université Félix Houphouët Boigny, en comparaison aux spécimens de l’herbier dudit centre.

\section{Traitement de données ethnobotaniques}

\section{Nomenclature}

La nomenclature des espèces de plantes de cette étude suit celle de Cronquist (1981). Les échantillons d’herbiers témoins sont déposés au C.N.F. 


\section{Fréquence de citations des espèces de plantes}

La fréquence de citations d'une espèce $(\mathrm{Fc})$ est le nombre de citations d'une espèce sur le nombre total de citations de toutes les espèces (Doh, 2015). A partir des informations recueillies auprès de vendeuses de plantes alimentaires sur les marchés, des maraîchers et des paysans interviewés, nous avons calculé la fréquence de citations de chaque espèce de plante selon la formule suivante indiquée par Fah et al (2013) :

Nombre de citations de l'espèce (n)

Fréquence de citations $(\mathrm{Fc})=$

Nombre total de citations de les toutes les espèces $(\mathrm{N})$

\section{Valeur d'usage thérapeutique (VUT)}

En ethnobotanique, la valeur d'usage permet de déterminer de façon significative les espèces ayant une grande valeur d'utilisation dans un milieu donné par rapport à d’autres espèces. Elle peut être calculée selon la formule simplifiée de Cotton (1996) suivante :

Nombre d'usages où l'espèce est mentionnée par chaque

Valeur d'Usage $(\mathrm{VU})=$ informateur (U)

Nombre d'informateurs ayant mentionné l'espèce $(\mathrm{N})$

Pour la VUT, le nombre d'usages correspond au nombre d'affections traité par une espèce donnée.

\section{Analyses statistiques}

Une analyse factorielle de correspondances (AFC) a été réalisée pour établir une relation entre l'abondance de chaque espèce de Corchorus et les localités visitées. Les plantes ont aussi été classées selon les deux paramètres (Fc et VUT) sur un même dendrogramme suivant la méthode de Ward, pour constituer des groupes homogènes.

\section{RESULTATS}

\section{Caractéristiques socio-démographiques des personnes enquêtées}

Au cours des enquêtes, 432 personnes ont été interrogées. Le nombre de personnes enquêtées varie d'une localité à une autre en fonction de l'abondance des plantes recherchées. Parmi ces personnes, on note 198 maraîchers (45,83\%), 150 vendeuses de plantes alimentaires (34,72\%) et 84 paysans $(19,45 \%)$. Ce sont des jeunes hommes et jeunes filles, des hommes et des femmes, dont l'âge varie entre 18 et 70 ans et ayant pour la majorité, le niveau d'étude primaire (figure 2). Ces interlocuteurs d’origines diverses, 
ont été rencontrés pour la plupart sur les sites maraîchers. Les femmes (95\%) sont majoritairement rencontrées dans les marchés alors que les hommes sont majoritaires dans les champs (80\%) et sur les sites maraîchers (67\%). Sur les sites maraîchers des différentes localités, on trouve une population d'autochtones minoritaires et une forte population de ressortissants de 4 pays de la l'Afrique de l’Ouest à savoir les Burkinabés, les Maliens, les Guinéens et les Togolais (figure 3). Les vendeuses des marchés et des maraîchers s'expriment tous en français malgré quelques difficultés liées au niveau d'instruction qui varie d'une personne à une autre. Pour la population paysanne, 72 sur 84 , soit $85,71 \%$ s'exprime en français et $14,29 \%$ ne parle que la langue locale.

\section{Espèces rencontrées et nomenclature traditionnelle}

L'étude a permis d'identifier quatre espèces du genre Corchorus utilisées en Côte d'Ivoire: Corchorus aestuans, Corchorus lobatus, Corchorus olitorius et Corchorus tridens. L’AFC réalisée (figure 4) explique à 77,68\% les corrélations entre l'abondance des espèces de Corchorus et les localités visitées. Elle fait apparaitre deux niveaux de corrélation. Le premier niveau concerne $C$. olitorius qu'on trouve plus à Man, à Guiglo, à Abidjan, à Bouaké et à Daloa. Le deuxième niveau concerne $C$. aestuans et $C$. lobatus qui apparaissent en abondance à Abengourou, à Bingerville, à Bouaflé, à Bondoukou, à Bouna, à Korhogo, à Touba et à Yamoussoukro. Cette analyse ne révèle pas de corrélation véritable entre les localités et $C$. tridens qu'on rencontre de façon rare à Katiola et à Odienné.

En rapport avec la nomenclature traditionnelle, les noms scientifiques des espèces répertoriées sont méconnus de toute la population interviewée. Les 4 espèces sont connues sous les mêmes appellations locales de Kplala, Kprala ou Kroala qui est d'origine Baoulé, le grand groupe ethnique du Centre. Le terme kroala, signifie littéralement « le pouvoir de se coucher ». Selon quelques investigateurs appartenant à ce groupe ethnique, ce nom a été donné à la plante pour dire que celui qui la consomme gagne en pouvoir c'est-à-dire en force physique. Les thermes kplala et kprala seraient des mauvaises prononciations ou des déformations du terme originel Kroala. Outre ce nom vernaculaire connu sur l'ensemble du territoire, chaque localité a son nom en fonction des groupes ethnolinguistiques rencontrés pendant l'enquête. Ces différents noms locaux sont représentés dans le tableau 1 en fonction des localités visitées. La différence entre ces 4 espèces se fait donc par les populations locales à partir de la coloration et de la forme des feuilles ou de l'aspect de la plante. Ainsi Corchorus lobatus est appelée sur le marché «Kroala fleur, Kroala feuilles courtes », C. olitorius est connue comme «Kroala rouge» et C.aestuans est nommée« Kroala blanc ». 
Corchorus tridens de couleur verte est aussi assimilée au «Kroala blanc ». Ici, la couleur blanche désigne la plante de coloration verte.

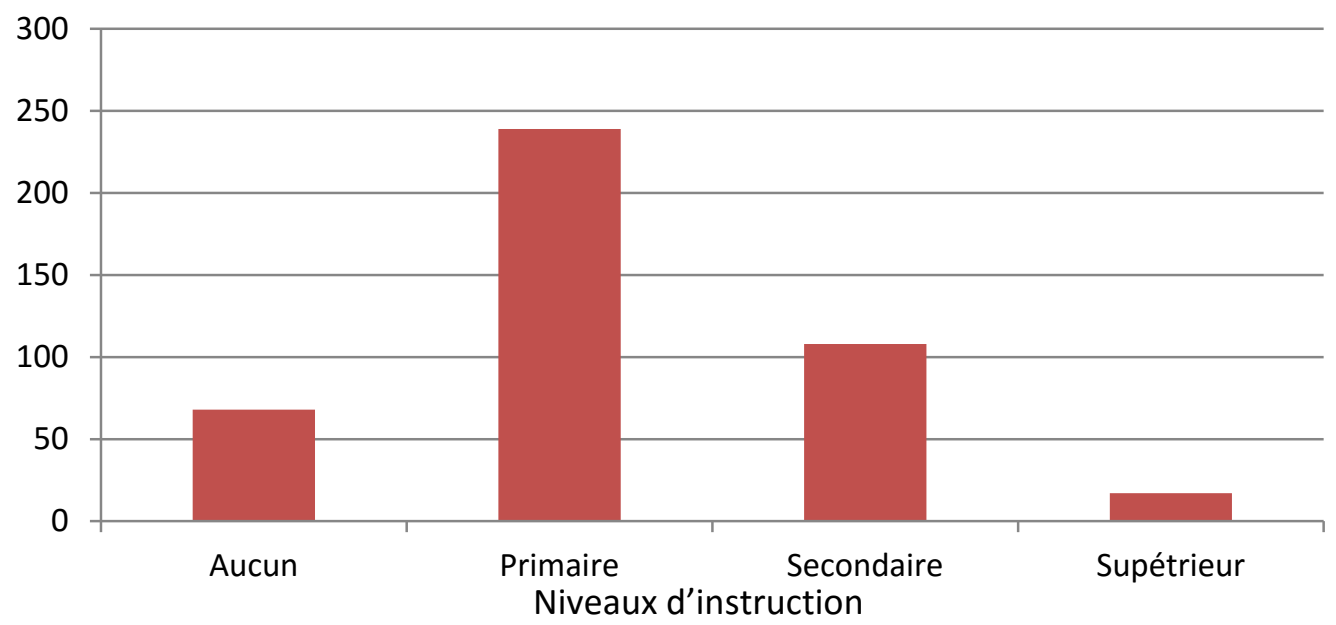

Figure 2 : Histogramme de répartition du nombre d'interviewés en fonction des niveaux d'étude

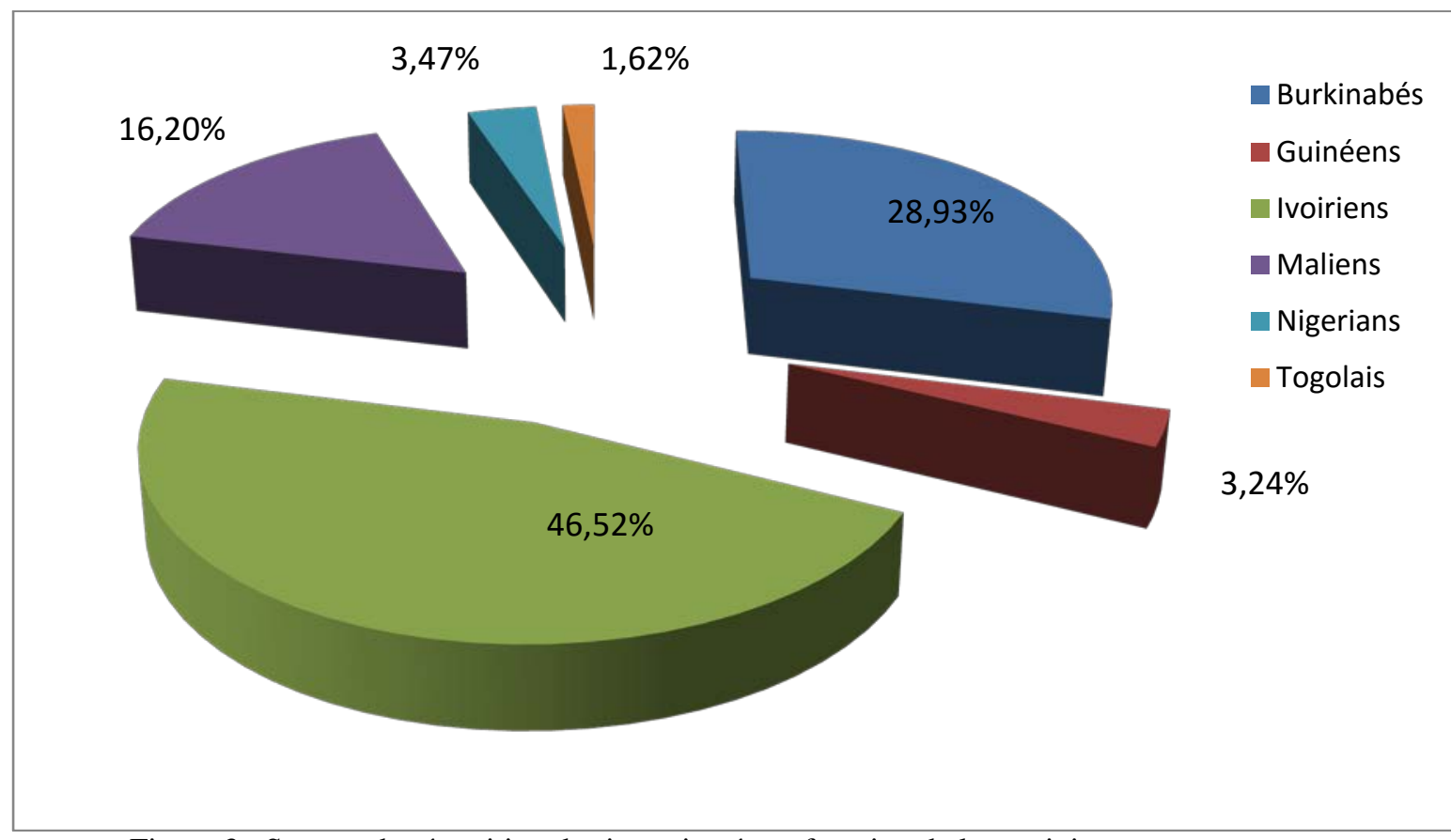

Figure 3 : Spectre de répartition des interviewés en fonction de leur origine 


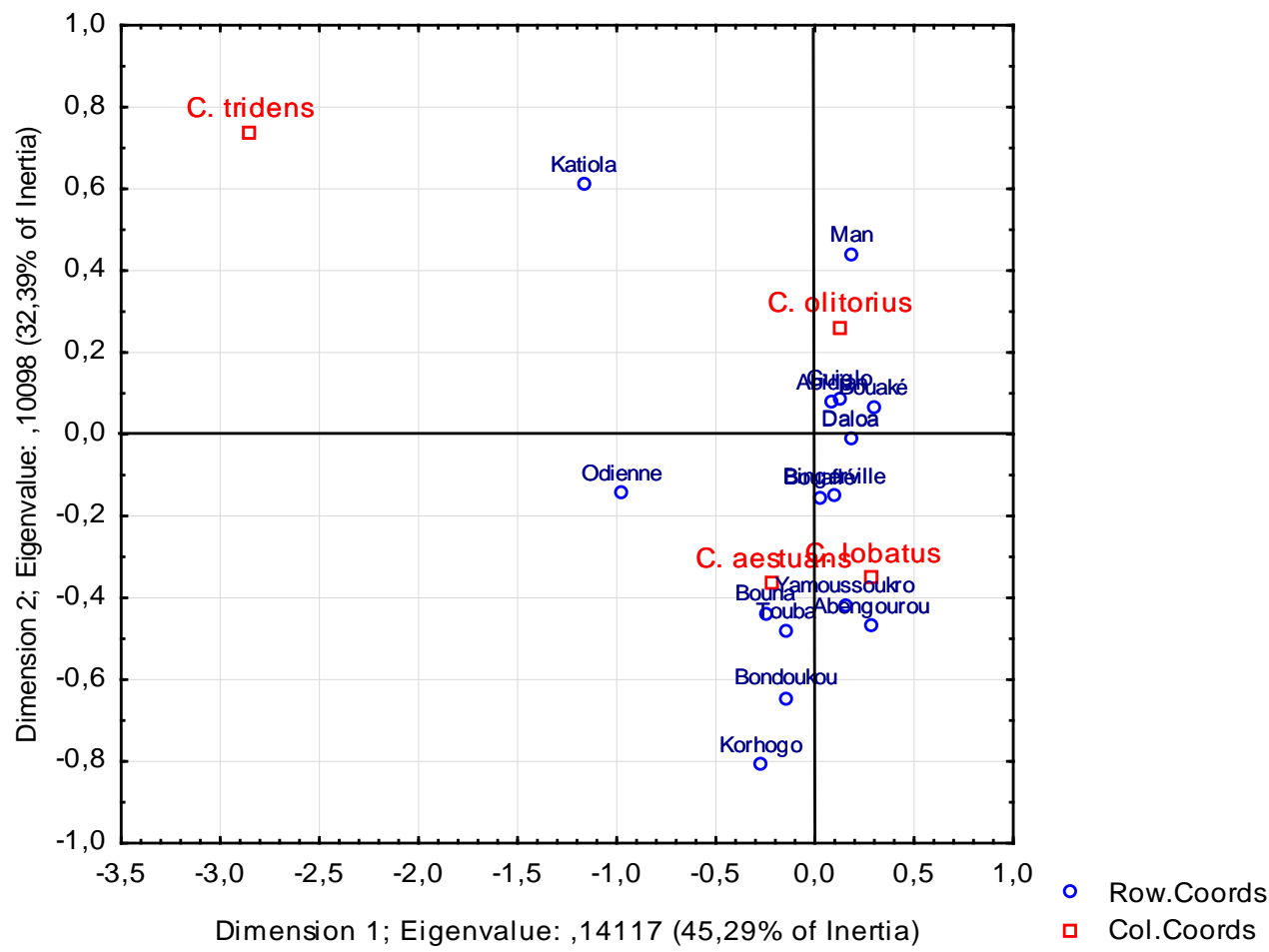

Figure 4 : Corrélations entre l'abondance des espèces du genre Corchorus et les localités visitées

Tableau 1: Noms vernaculaires de la plante selon le groupe ethnolinguistique

Tableau 1 (Suite) : Noms vernaculaires de la plante selon le groupe ethnolinguistique

\begin{tabular}{|l|l|l|}
\hline $\begin{array}{l}\text { Groupes ethnolinguistiques } \\
\text { rencontrés }\end{array}$ & $\begin{array}{l}\text { Noms vernaculaires } \\
\text { attribués à la fois aux 4 } \\
\text { espèces indiquées }\end{array}$ & $\begin{array}{l}\text { Localités } \\
\text { (massif) } \\
\text { du groupe }\end{array}$ \\
\hline Abron & Assuepriwa & Bondoukou \\
\hline Agni & Kprala & Abengourou \\
\hline Baoulé & Kprala & Bouaké, Yamoussoukro \\
\hline Bété & Zrôô ou Zrôhô & Daloa \\
\hline Ébrié & Nasrin & Abidjan \\
\hline Gouro & Bianin & Bouaflé \\
\hline Guéré & Srin & Guiglo \\
\hline Lobi & Bantchôlô & Bouna \\
\hline Maouka & Nabia & Touba \\
\hline Mounan & Gbôhô & Bouaflé \\
\hline
\end{tabular}




\begin{tabular}{|l|l|l|}
\hline Odieneka & Zonbrôô & Odienné \\
\hline Mounan & Gbôhô & Bouaflé \\
\hline Odieneka & Zonbrôô & Odienekas \\
\hline Sénoufo & Gbô-houor & Korhogo \\
\hline Tagbana & Djoflon & Katiola \\
\hline Wobé & Srè & Guiglo \\
\hline Yacouba & Plôhô & Man \\
\hline Burkinabés & $\begin{array}{l}\text { Blouvaka (Mossi), Djintara } \\
\text { (Bissa), Vantchôlô (Moré), }\end{array}$ & $\begin{array}{l}\text { Dans toutes les localités } \\
\text { visitées }\end{array}$ \\
\hline Guinéens & Kôlor (Gblèssè) & Abidjan, Bouaké \\
\hline Maliens & Sofèbourou (Bambara) & $\begin{array}{l}\text { Abidjan, } \\
\text { Bouaké, } \\
\text { Daloa, Guiglo, Man } \\
\text { Abidjan }\end{array}$ \\
\hline Nigerians & Obidjan \\
\hline Togolais & Owô (Yorouba) \\
\hline
\end{tabular}

\section{Usages des espèces rencontrées}

Les quatre espèces du genre Corchorus rencontrées sont toutes des plantes utilisées dans le domaine de l'alimentation et de la médecine traditionnelle.

\section{Usage alimentaire des espèces du genre Corchorus}

Les plantes du genre Corchorus ont été rencontrées dans toutes les localités où elles sont consommées. Les feuilles de ces espèces sont utilisées à l'état frais et sec pour faire des sauces ou des pâtes accompagnant des mets. Les plats varient en fonction des groupes ethniques. Ainsi, chez les Baoulé, la sauce «Kroala » se mange fréquemment avec le placali. Chez les Yacouba originaires de Man, la pâte de Kroala grillé accompagne le riz alors que la sauce se consomme avec du kongodé (placali noir fait à base de poudre de manioc séché). Chez les Gouro, la sauce se mange surtout en saison pluvieuse avec le foutou igname.

\section{Usage ethnomédicinal}

Sur une population de 432 enquêtés, seulement 104 personnes (24,07\%) ont renseignés sur les vertus thérapeutiques des plantes.

Plantes et affections traitées

Les affections citées par les enquêtés, la partie de la plante utilisée, le mode de préparation du médicament, la forme médicamenteuse et le mode d'administration sont consignés dans le tableau 2.

Parties des plantes utilisées comme drogue

Plusieurs parties des espèces sont utilisées dans la préparation des phytomédicaments : tiges feuillées, feuilles, graines et racines. Les feuilles $(53,57 \%)$ sont majoritairement sollicitées. Les racines sont utilisées à 
$25 \%$ des cas. Les plantes entières, les graines, et les tiges sont sollicitées dans les recettes médicamenteuses respectivement dans $10,71 \% ; 7,14 \%$ et $3,57 \%$ des cas. Ces différentes valeurs sont indiquées par la figure 5 .

Modes de préparation des médicaments

Les investigations révèlent plusieurs modes préparation des phytomédicaments: décoction, incinération, pétrissage, pilage, cuisson et trituration. La décoction est le mode de préparation la plus importante avec 53,57\% des cas. Le pétrissage est le second mode de préparation sollicité $(17,85 \%)$.

Formes médicamenteuses et modes d'administration

En rapport avec les formes médicamenteuses, on obtient plusieurs cas : le décocté, la pâte, la poudre, le triturât et la sauce. Le décocté est le plus sollicité avec $53,84 \%$ des cas. Divers modes d'administration ont été enregistrés : boisson, pâte à consommer, application locale, lavement (purge) et instillation nasale. La boisson est le mode d'administration le plus fréquent dans $50 \%$ des cas. L'application locale est aussi bien pratiquée avec 28,57\%. L'absorption (10,71\%), le lavement (7,14\%) et l'instillation nasale (3,57\%) sont peu sollicités.

Spécificité de recettes médicamenteuses

Les investigations révèlent des recettes médicamenteuses préparées seulement à partir d'organes de plantes. La phytothérapie se distingue ici de l’opothérapie et de la minéralothérapie. 
Tableau $2:$ Plantes du genre Corchorus et indications thérapeutiques

\begin{tabular}{|c|c|c|c|c|}
\hline Affection & Espèce & Partie utilisée & Mode de préparation & Mode d'administration \\
\hline \multirow{2}{*}{ Anémie - } & C. lobatus & Feuilles & Cuisson : pâte & Consommation \\
\hline & C. olitorius & Fevilles & Cuisson : pate & Consommation \\
\hline Anthrax & C. olitorius & Racines & Pétrissage : pâte & Application locale \\
\hline Asthme & C. tridens & Graines & Pétrissage avec FOg : pâte & Instillation nasale \\
\hline Blessure & C. olitorius & Feuilles & Trituration : triturât & Application locale \\
\hline Bouton de V. & C. tridens & Graines & Pilage : poudre $+\mathrm{BK}$ & Application locale \\
\hline Brûhure & C. aestuans & Racines & Incinération : cendre $+\mathrm{HP}$ & Application locale \\
\hline Cardiopathie & C. olitorius & Fevilles & Décoction : décocté & Boisson \\
\hline Cervicalgie & C. aestuans & Racines & Incinération : cendre $+\mathrm{HP}$ & Application locale \\
\hline Constipation & C. olitorius & Feuilles & Cuisson : pâte & Consommation \\
\hline Diabète & C. olitorius & Feuilles & Décoction : décocté & Boisson \\
\hline Diarrhée & C. olitorins & Feuilles & Décoction : décocté & Boisson \\
\hline Douleurs p. & C. olitorius & Feuilles & Décoction : décocté & Boisson \\
\hline Dysenterie & C. olitorius & Racines & Décoction : décocté & Boisson \\
\hline Enfluxes & C. aestuans & Racines & Pétrissage avec PL : pâte $+E$ & Purge \\
\hline Fatigue gle & C. olitorius & Feuilles & Décoction : décocté & Boisson \\
\hline Fièvre jaune & C. tridens & Feuilles & Décoction : décocté & Boisson \\
\hline Fièvre typhoide & C. olitorius & Plante ent. & Décoction : décocté+ JC & Boisson \\
\hline Goitre & C. olitorius & Racines & Incinération : cendre+ $\mathrm{HP}$ & Application locale \\
\hline Hématurie & C. tridens & Plante ent. & Décoction : décocté & Boisson \\
\hline Hypertension & C. olitorius & Feuilles & Décoction : décocté & Boisson \\
\hline Hypotension & C. olitorius & Feuilles & Décoction : décocté & Boisson \\
\hline Kwashiorkor & C. aestuans & Feuilles & Décoction : décocté & Boisson \\
\hline Maux de ventre & C. aestuans & Racines & Pétrissage avec $G G$ : pâte $+E$ & Purge \\
\hline
\end{tabular}

Signification des symboles :

C. : Corchorus

Douleurs P. : Douleurs persistantes

Plante ent. : Plante entière

FOg : Feuilles de Ocimum gratissimum E : Eau girofle
Boutons de V.: Boutons de visage HP : Huile palmiste

Fatigue gle : Fatigue générale

BK : Beurre de karité

JC : Jus de citron

PL : poivre long GG : Grains de 
Sur 28 recettes exposées dans le traitement de 27 affections, une seule recette est bispécifique et les 27 autres sont monospécifiques. Les recettes plurispécifiques n’ont pas été indiquées. Les recettes monospécifiques sont donc largement majoritaires avec $96,43 \%$ contre $3,57 \%$ pour la recette bispécifique.

Fréquence de citations et valeur d'usage thérapeutique

Les fréquences de citation et valeurs d'usages thérapeutiques ont permis de faire une classification hiérarchique des espèces de Corchorus (figure 6). Le dendrogramme obtenu montre trois groupes de plantes lorsqu'on réalise une coupe à la distance euclidienne de 0,5. Le premier groupe est constitué de Corchorus olitorius, l'espèce la plus citée et la plus utilisée en thérapie ( $\mathrm{Fc}=0,55 ; \mathrm{VUT}=3.06)$. Le deuxième groupe est composé de Corchorus lobatus $(\mathrm{Fc}=0,03$; VUT=1). Corchorus aestuans $(\mathrm{Fc}=0,24$; $\mathrm{VUT}=3,06)$ et Corchorus tridens $(\mathrm{Fc}=0,17$; VUT=1,63) forment le troisième groupe.

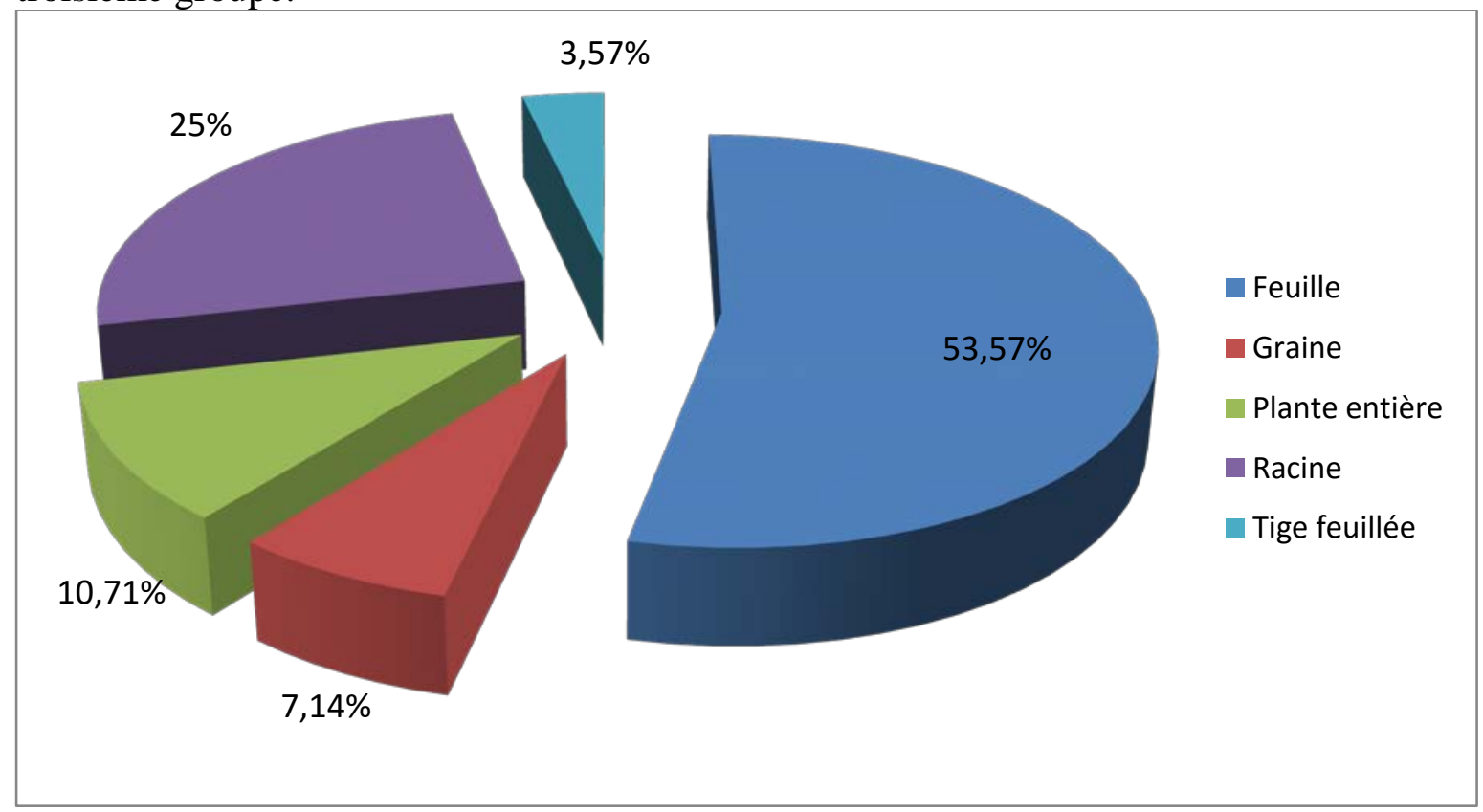

Figure 5: Spectre de répartition des parties de la plante utilisée comme drogues 
Tree Diagram for 4 Cases

Ward's method

Squared Euclidean distances

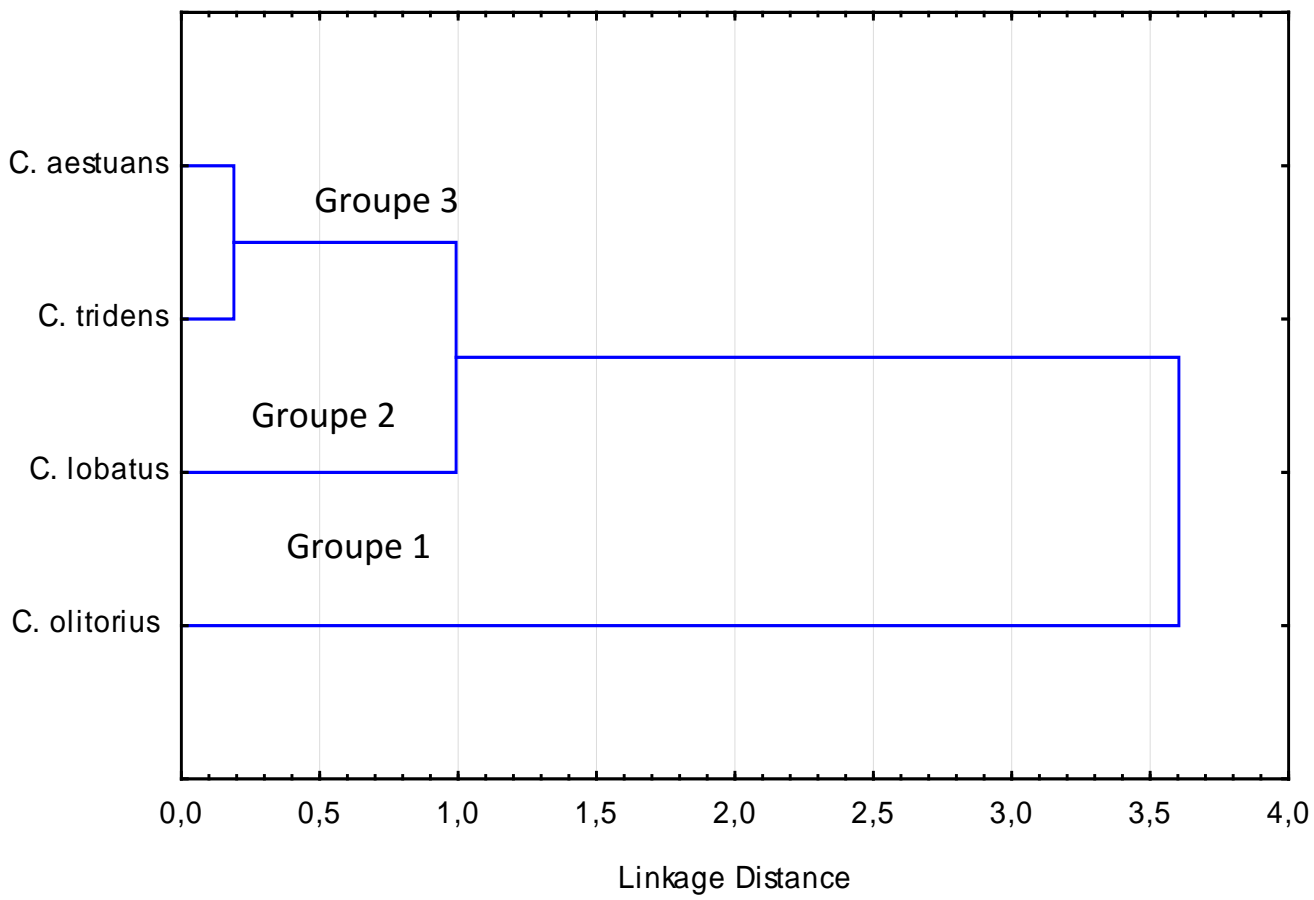

Figure 6 : Dendrogramme de la classification hiérarchique des espèces recensées en fonction de la fréquence de citation ( $\mathrm{Fc}$ ) et de la valeur d'usage thérapeutique (VUT)

\section{DISCUSSION}

\section{Caractéristiques socio-démographiques des personnes enquêtées}

Les espèces du genre Corhorus existent dans les 15 localités visitées. Cela montre bien que ces plantes sont cultivées sur toute l'étendue du territoire ivoirien. On rencontre dans chaque localité une population cosmopolite qui s'intéresse à la culture de ces plantes. Ce sont les ivoiriens (les autochtones et allogènes venant d'autres régions du pays) qui représentent $46,52 \%$ et les non nationaux ressortissants de pays de la CEDEAO (53,48\%). Ce constat montre bien le brassage interethnique en Côte d'Ivoire. Parmi ces étrangers, les Burkinabés sont majoritaires. Les interviewés ont majoritairement un niveau d'étude primaire $(55,32 \%)$. Ce résultat est proche de celui de Aké (2015) qui a estimé à 44\%, la proportion des personnes interrogées ayant ce même niveau d'étude, dans son étude sur les plantes et champignons alimentaires dans le Département d' Agboville et dans le District d' Abidjan. Au sein de la population enquêtée (vendeuses de plantes, maraîchers et paysans), les maraîchers sont majoritairement 
représentés. Ce résultat montre que les plantes du genre Corchorus vendues sur les marchés proviennent pour la plupart des sites maraîchers.

\section{Espèces rencontrées et nomenclature traditionnelle}

L’abondance des quatre espèces du genre Corchorus répertoriées varie d’une localité à une autre. Cela pourrait s'expliquer par la variation des préférences alimentaires en fonction des groupes ethniques (Aké-Assi E., 2015).

En rapport avec la nomenclature traditionnelle, il est fréquent qu'une même espèce porte plusieurs noms locaux ou que plusieurs plantes portent le même nom (Doh, 2015). Cette étude fait le même constat. En effet, les 4 espèces rencontrées portent le même nom : Kroala. Ce nom propre à un groupe ethnique a été adopté dans toutes les localités visitées. Cependant, sur les marchés, la différence de coloration et la forme des feuilles sont utilisées pour distinguer les espèces. Les adoptions de noms d'un groupe sont bien connues dans le domaine de la médecine traditionnelle où les noms des plantes sont donnés sur les marchés en langue malinké (Ta-Bi, 2013). Cette adoption tend à faire méconnaitre les noms locaux aux jeunes générations. La nomenclature traditionnelle reste donc imprécise et incomplète. Le manque de données écrites représente un problème majeur à travers le continent ou même au sein d'une ethnie donnée (Doh, 2015).

\section{Usages des espèces rencontrées}

\section{Usages culinaires des espèces rencontrées}

Dans l'alimentation, seules les feuilles des espèces identifiées par cette étude sont utilisées. Ces résultats sont proches de ceux de Kouamé (2009) qui a révélé dans son étude relative aux plantes alimentaires de Gagnoa (au Centre-Ouest de la Côte d'Ivoire) que les mêmes organes sont employés majoritairement à 34\%. Par ailleurs, nos résultats montrent que la consommation des plantes du genre Corchorus est pratiquée chez plusieurs groupes ethnolinguistitiques du pays.

\section{Usages des espèces rencontrées en médecine traditionnelle Plantes et affections traitées}

Les investigations ethnobotaniques indiquent une liste de 27 pathologies qui sont traitées par les plantes du genre Corchorus en Cote d'Ivoire. On retrouve dans cette liste plusieurs affections telles que l'anémie, la douleur, la dysenterie, la toux, le kwashiorkor, l'insuffisance cardiaque, la constipation et diarrhée qui sont déjà évoquées au Sénégal (Mbaye 2002) et au Nigeria Osawaru et al. (2012). Cette étude confirme les indications thérapeutiques déjà évoquées par ces auteurs. 


\section{Parties des plantes utilisées comme drogue}

Les feuilles sont majoritairement sollicitées dans la préparation des recettes médicamenteuses avec $53,57 \%$. Ce résultat est très proche de celui Doh (2015) qui a indiqué que les feuilles étaient sollicitées, majoritairement, dans 56,89 \% des cas. Il est aussi proche de celui de AkéAssi E. (2015) qui a révélé l'utilisation des feuilles à $41,86 \%$ des cas, dans son étude sur les plantes à potentialité décorative de la flore du sud de la Côte d'Ivoire.

\section{Mode de préparation des médicaments}

Cette étude a indiqué 6 techniques de préparation médicamenteuse: décoction, incinération, pétrissage, pilage, cuisson et trituration. Parmi ces modes de préparation identifiés, la décoction est la plus utilisée (50\%). C’est un résultat proche de celui de Konkon (2009) qui a indiqué que la décoction est majoritairement utilisée dans $75 \%$ des cas, dans son étude relative aux plantes antidiabétiques vendues sur les marchés d'Abidjan (Côte d'Ivoire).

\section{Modes d'administration et forme médicamenteuse}

Selon nos investigations, la boisson est le mode d'administration le plus fréquent avec 52\% des cas. Ce résultat est proche de celui de N'Guessan et al. (2009) qui ont indiqué ce taux à 48,97\% des cas.

$\mathrm{Au}$ niveau des formes médicamenteuses, le décocté est le plus sollicité avec 53,84\% des cas. Ce résultat est proche de celui de Doh (2015) qui a indiqué que le décocté est majoritairement utilisé à 82,35\% des cas. Il est contraire à celui de N'Guessan (2008) qui a révélé les pâtes comme formes médicamenteuses les plus sollicitées chez les peuples Abbey et Krobou d'Agboville (Côte d'Ivoire).

\section{Fréquence de citations (Fc) et valeur d'usage thérapeutique (VUT)}

L'espèce Corchorus olitorius a les valeurs de Fc et VUT les plus importantes. Elle est donc la plante la plus utilisée par les populations interviewées. Cela pourrait s'expliquer par le fait que cette espèce est très connue et surtout beaucoup consommée Aké et al. (2015).

\section{CONCLUSION}

Les investigations ethnobotaniques, dans les 15 localités de la Côte d'Ivoire, ont permis de recenser 4 espèces du genre Corchorus. Elles sont consommées et utilisées en médecine traditionnelle. Les feuilles de ces plantes nommées vulgairement Kroala sont consommées sur l'étendue du territoire en sauce accompagnant plusieurs mets. Ces espèces sont cultivées à la fois par les paysans et les maraîchers. Au plan 
ethnomédicinal, ces espèces interviennent dans le traitement de plusieurs pathologies. Diverses parties de ces plantes: feuilles, racines, tiges feuillées, et graines sont employées pour préparer des recettes médicamenteuses majoritairement monospécifiques. Pour ces nombreux apports, la culture de ces plantes du genre Corchorus doit être encouragée et vulgarisée en Côte d’Ivoire.

\section{References:}

Aiyeroja A. A. and Bello O. A., 2006. Ethnobotanical potentials of common herbs in Nigeria: A case study of Enugu state Educational Research and Review 1 (1): 16-22..

Aké-Assi E., 2015. Plantes à potentialité décorative de la flore du sud de la Côte d'Ivoire : études taxinomique, ethnobotanique et essai de domestication de Thunbergia atacorensis (Acanthaceae), une espèce nouvellement introduite. Thèse de Doctorat d'Etat ès Sciences Naturelles, Spécialité Ethnobotanique, Université de Cocody-Abidjan (Côte d'Ivoire), UFR Biosciences, Laboratoire de Botanique, 219 p.

Aké C. B., 2015. Etude ethnobotanique des plantes et des champignons spontanés, utilisées en alimentation dans le département d'Agboville et le District d'Abidjan (Côte d'Ivoire). Thèse de Doctorat de l’Université Félix Houphouët Boigny de Cocody-Abidjan (Côte d'Ivoire), UFR Biosciences, 181 p.

Aké C. B., N’Guessan K., Kouamé N.M., 2015. Traditional Consumption Stateous of Wild Food Plants and Mushrooms Species in Abidjan and Agboville (Côte d'Ivoire). European Journal of Scientific Research Vol. 135 No 1: 182-195

Cronquist A., 1981.An integrated System of Classification of flower plants. Colombia University, press of New York (US), 1262 p.

Doh K. S., 2015. Plantes à potentialité antidiabétique utilisées en médecine traditionnelle dans le District d'Abidjan (Côte d'Ivoire): étude ethnobotanique, caractérisation tri phytochimique et évaluation de quelques paramètres pharmacodynamiques de certaines espèces. Thèse de Doctorat de l'Université Félix Houphouët Boigny de Cocody-Abidjan (Côte-d’Ivoire), UFR Biosciences, 150 p.

Fah L., Klotoé J.R., Dougnon V., Koudokpon H., Fanou V.B.A., Dandjesso C. et Loko F., 2013 : Etude ethnobotanique des plantes utilisées dans le traitement du diabète chez la femme enceinte à Cotonou et Abomey-Calali (Benin). Journal of Animals et plant Sciences 18(1) : 2647-2658.

Guy Lalière, 2009. Plantes comestibles, cueillette et recettes des 4 saisons. Edition Debaisieux, 174 p.

Konkon N. G., 2009. Recensement de plantes préconisées comme antidiabétiques par les vendeuses de plantes sur les marchés d’Abidjan: 
évaluation de l'activité anti-diabétique des feuilles de Mitragyna inermis (Rubiaceae). Thèse de Doctorat de l’Université Félix Houphouët Boigny de Cocody-Abidjan (Côte-d'Ivoire), UFR Biosciences, 168 p.

Kouamé N.M.T., 2009. Espèces végétales spontanées consommées dans le Département de Gagnoa, au Centre-Ouest de la Côte d'Ivoire : inventaire, teneur en quelques nutriments et essais de domestication. Doctorat de Botanique, U.F.R. Biosciences, Université de Cocody-Abidjan, Côte d'Ivoire, $140 \mathrm{p}$.

Mbaye M. S., 2002. Contribution à l'étude biosystématique du genre Corchorus L. (Tiliacea) au Sénégal, Thèse de Doctorat, université cheikh antadiop de dakar, $134 \mathrm{p}$.

N’Guessan K. 2008. Plantes médicinales et pratiques médicales traditionnelles chez les peuples Abbey et Krobou du Département d'Agboville (Côte d'Ivoire). Thèse de Doctorat d'Etat ès Sciences Naturelles, Spécialité Ethnobotanique, Université de Cocody-Abidjan (Côte d'Ivoire), UFR Biosciences, Laboratoire de Botanique, 235 p.

N’Guessan K.,Kadja B., Zirihi G.N., Traoré D.et Aké-Assi L., 2009. Screening phytochimique de quelques plantes médicinales ivoiriennes utilisées en pays Krobou (Agboville,Côte d'Ivoire). Science \& Nature, 6 (1) : $1-15$

N’Guessan K., Kouamé N.M.T., Assi-Kaudjhis C. et Aké C.B., 2015. Ethnobotanical study of spontaneous wild plants used for food by Krobou people, in the south of Côte d'Ivoire. Journal of Global Biosciences, Volume 4, Number 2: 1354-1365

Osawaru, M. E., Ogwu, M. C., Chime, A.O. and Amorighoye, A. (2012): Morphological evaluation and protein profiling of three accessions of nigerian corchorus linn. Species. Bayero. Journal of Pure and Applied Sciences, 5 (1): 26 - 32

RGPH, 2014. Recensement Général de la Population et de l'Habitat, Résultats globaux. Secrétariat Technique Permanent du Comité Technique du RGPH, Côte d’Ivoire, 26 p.

Ta-Bi I.H., 2013. Etude ethnobotanique des plantes antidiabétiques vendues sur les marchés de la commune de Yopougon dans le district d’Abidjan (Cote d'ivoire). D.E.A. d'Ecologie tropicale. Université de Félix Houphouët Boigny d'Abidjan (Cote d'ivoire), UFR Biosciences, 46 p.

Tahiri N., El -Basty A., Zidane L., Rochdi A., Douira A., 2012. Etude ethnobotanique des plantes médicinales dans la Province de Settah (Maroc), Université Ibn Tofail. Laboratoire de Botanique et de protection des plantes. Faculté des Sciences. Kastamonu Uni., Orman Fakultesi Dergisi, Journal of forestry faculty, 12(2): 192-208. 